\title{
Finite element modelling of air cavities effect on GnP/epoxy nanocomposite thermal response and its full-field validation
}

\author{
Asimina Manta ${ }^{1}$, Matthieu Gresi $1^{1,2}$, and Constantinos Soutis ${ }^{2 *}$ \\ ${ }_{1}$ i-composites lab, School of Materials, University of Manchester, James Lighthill Building, Sackville \\ Street, Manchester, M19 9PL, UK \\ ${ }^{2}$ Aerospace Research Institute, Faculty of Science and Engineering, University of Manchester, James \\ Lighthill Building, Sackville Street, M13 9PL, UK
}

\begin{abstract}
The manufacturing process of $\mathrm{GnP} /$ polymer nanocomposites exhibits important challenges regarding the material quality and how to reduce or eliminate fabrication induced defects. In this work, the effect of air cavities (voids) on the thermal response of nanocomposites is studied by means of finite element analysis on a multi-scale scheme. The proposed model is validated with experimental data by performing generic and fullfield comparisons.
\end{abstract}

\section{Introduction}

The manufacturing process of any composite material system exhibits important challenges, including the development of time and cost-effective methods, enabling massive production with high repeatability in the products' specification. In terms of structural integrity, defects are induced during the fabrication process. The solid structures shrinkage, particle contamination, non-uniform particle distribution and trapped air are considered the most common and crucial issues, as they affect the performance and the life cycle of the composite component. The air trapped in the form of bubbles of various sizes is one of the most common defect encountered especially in the manufacturing of nanocomposites of high weight (or volume) fraction. The high viscosity of the nanofluid does not allow the air to be removed efficiently, while the increase of degassing temperature is limited by the polymerisation temperature and the polymer pot life. Therefore, it is necessary to eliminate or reduce the void content but more important to quantify its effect on the thermomechanical response.

In this work, a 3D multi-scale finite element model, based on previous research [1-4], is presented to simulate the steady-state and transient thermal response of a graphene nanoplatelet $(\mathrm{GnP}) /$ epoxy composite in the presence of surface air cavities. It adopts a Representative Volume Element (RVE), modelling the effect of the cavity on the local thermal diffusivity field, and the square specimen for thermal diffusivity measurements. The composite samples were analysed macroscopically, obtaining both effective thermal

*Corresponding author: constantinos.soutis@manchester.ac.uk 
properties and their mapping. The simulated macroscopic and field thermal response were compared with experimental measurements obtained for a 10wt\% M25 GnP/epoxy nanocomposite with surface impurities, while the use of the thermal diffusivity mapping to locate air bubble defects was explored.

\section{Experimental procedures}

\subsection{Materials}

For the sample preparation, GradeM25 GnP graphene nanoplatelets acquired by XG Sciences [5] were used in the form of powder form. The nanoparticles exhibited an average thickness of $6-8 \mathrm{~nm}$ and an average length of $25 \mu \mathrm{m}$ as it was shown in SEM image (Fig. 1(a)) taken on the fracture surface of the specimen. The specific surface area was in the region of $120-150 \mathrm{~m}^{2} / \mathrm{g}$. The nanoparticles were combined with a polymeric matrix made of a low-viscosity bisphenol-A epoxy resin (Araldite LY564) and a cycloaliphatic polyamine curing agent (Aradur 2954) with a mixing ratio of 100:35 (epoxy: hardener) supplied by Huntsman [6].

\subsection{Manufacturing Process}

At first, the graphene powder was added to acetone, forming a solution of a concentration of $50 \mathrm{mg} / \mathrm{ml}$ and put on a sonication bath for 30 minutes to dissolve the particles stacks. Afterwards, the liquid epoxy was added to the solution, which was shear mixed at 3000rpm for 1 hour while heated at $80^{\circ} \mathrm{C}$ to decrease the viscosity. By the end of the process, the presence of residual acetone was checked and any remains were evaporated by hot-stirring of the mixture at $80^{\circ} \mathrm{C}$. Then, the mixture was let to reach the room temperature, hardener was added, followed by stirring for 5 minutes at $1000 \mathrm{rpm}$. After degasification, the mixture was transferred to a silicone rubber mould with the aid of a syringe. The material was cured at $80^{\circ} \mathrm{C}$ for 2 hours and post-cured at $140^{\circ} \mathrm{C}$ for 8 hours. The obtained specimens were $60 \times 60 \mathrm{~mm}^{2}$ plates with an average thickness of $6 \mathrm{~mm}$. All the specimens were surface grinded to obtain flat surfaces and uniform specimen thickness. After surface grinding, the presence of air cavities was observed, suggesting that complete degasification of the $10 \mathrm{wt} \%$ loaded specimen didn't happen due to the relatively high viscosity of the material.

\subsection{Thermal Diffusivity Mapping}

The thermal diffusivity mapping was obtained by the flash method in accordance with the standard ASTM E1461 [7]. Infrared (IR) thermography was used to measure the thermal diffusivity $(\alpha)$ of the sample. An external heat source (two flash lamps, delivering a combined pulse of approximate $6 \mathrm{~kJ}$ over duration of $10.6 \mathrm{~ms}$ ) heated the front face of the sample, while an IR camera (Thermosensorik GmbH, Germany) was recording the thermal radiation (temperature) of the rear surface for each pixel. The thermal diffusivity was calculated for each point (pixel) of the field in accordance with the procedure described in [7], [8], once the signal of each point was de-noised numerically. Especially, the timedependent response of each pixel was recorded and de-noised with an 8-grade symlet wave function performing a 6-level wavelet decomposition. The thermal diffusivity mapping obtained for the $10 \mathrm{wt} \% \mathrm{GnP} /$ epoxy nanocomposite is presented in Fig. 1(b). Each pixel corresponds to length of $100 \mu \mathrm{m}$. 

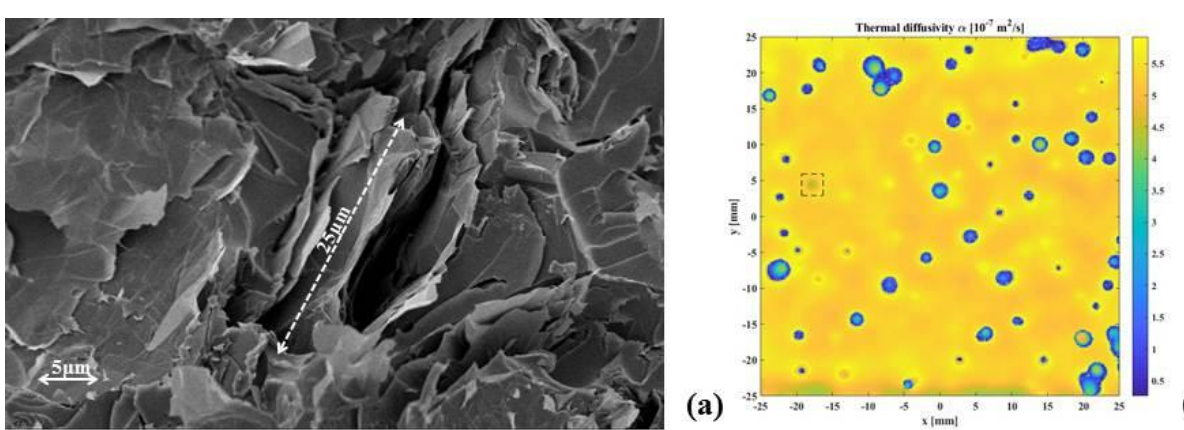

(b)

Fig. 1. (a) SEM image on the fracture surface of $10 \mathrm{wt} \% \mathrm{GnP} /$ epoxy nanocomposite and (b) thermal diffusivity mapping of $10 \mathrm{wt} \% \mathrm{GnP} /$ epoxy nanocomposite illustrating air cavities.

\section{Numerical Analysis}

\subsection{Air Cavities Mapping}

The temperature fields obtained during the experiment for the thermal diffusivity mapping was used to identify the air cavities. The cavities were easily detected in the temperature field during the transient response by the significantly lower temperature compared to the rest temperature field as it could be noted in Fig. 2(a). A snapshot of the temperature field was appropriately chosen so as the cavities could be noted on their initial size and shape before the temperature field evolved around them. In our case, the snapshot at time $0.25 \mathrm{sec}$ was selected. The temperature field was made dimensionless and a threshold of 0.69 was selected such that all the pixels with values below that threshold may represent the cavities. In this stage, both surface and internal voids could be distinguished. The surface cavities could be easily recognised in the thermal diffusivity map by significantly lower thermal diffusivity in their perimeter as a result of the edge effect. In Fig. 2(b), the mapping of the cavities is depicted. Analysing the mapping of imperfections, it was found that void volume fraction is $3.07 \%$. 


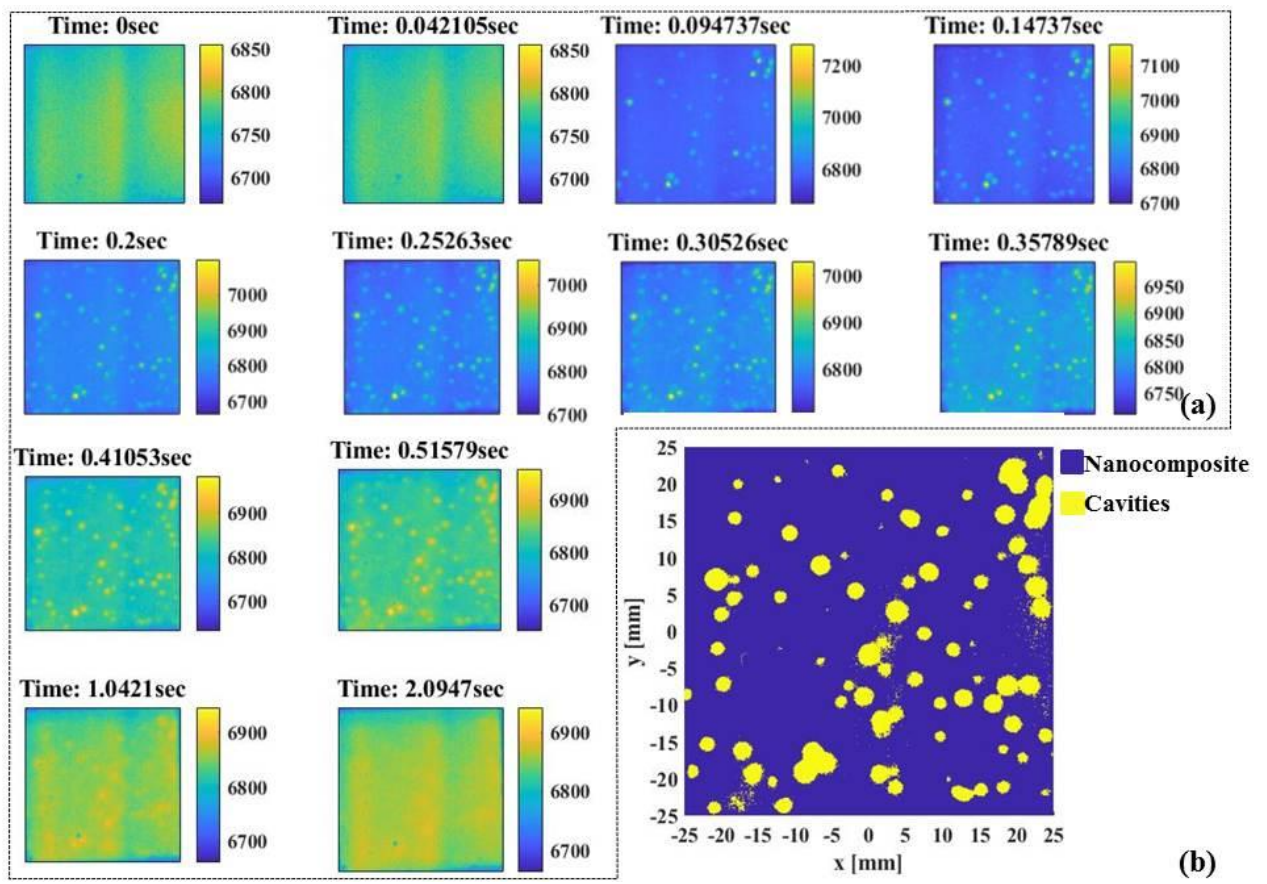

Fig. 2. (a) Evolution of temperature field as function of time and (b) mapping of void content for the $10 \mathrm{wt} \% \mathrm{GnP} /$ epoxy specimen

\subsection{Multi-Scale Finite Element Model}

\subsubsection{Effective thermal response}

The 3D multi-scale finite element model, based on previous research [1-4], consists of a Representative Volume Element (RVE), modelling the effect of the cavity on the local thermal diffusivity field, and a square specimen for full-field thermal diffusivity measurements. The finite element models were built in the commercial FE package ANSYS 16.2 with 8-node 3D thermal solid element SOLID87. The RVE consists of a sphere-air bubble- with diameter $\mathrm{d}$ and the thermal properties of air at room temperature, surrounded by nanocomposite material in cubical shape (Fig. 3(d)). The equivalent structure was analysed, obtaining effective thermal properties (conductivity and diffusivity). In Table 1, the material properties used for the RVE analysis are summarised.

The parametric study conducted on the RVE showed that the size of the air cavity did not affect the thermal response for a specific volume fraction as it is depicted in Fig. 3(a). However, the increase in void content results in a decrease in the nanocomposite thermal response (Fig. 3(b) and (c)). The effective thermal diffusivity obtained was found equal to the experimental average thermal diffusivity (Fig. 3(c)). 

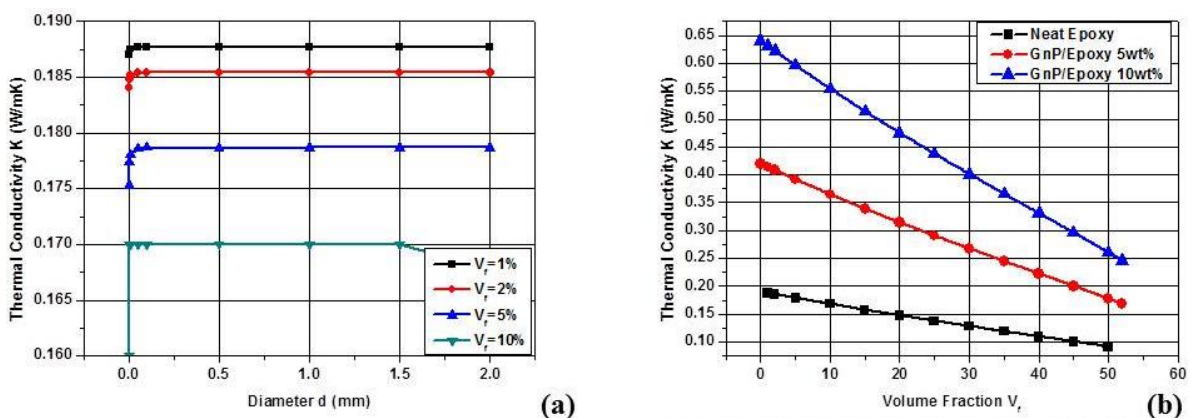

(a)
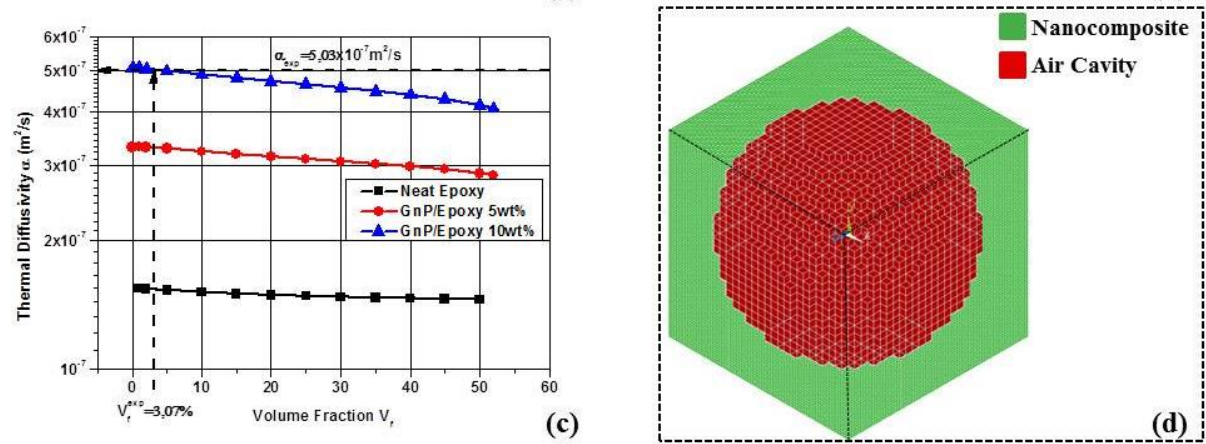

Fig. 3. (a) Effect of air cavity diameter on the thermal conductivity of pure epoxy for a range of void volume fraction (1\%-40\%), (b) effect of void volume fraction on thermal conductivity, (c) thermal diffusivity of pure epoxy, $5 \mathrm{wt} \%$ and $10 \mathrm{wt} \% \mathrm{GnP} /$ epoxy nanocomposite and (d) unit cell FE model.

Table 1. Material properties used in RVE model.

\begin{tabular}{|c|c|c|c|c|}
\hline \multirow{2}{*}{ Phase } & \multicolumn{4}{|c|}{ Thermal Properties } \\
\cline { 2 - 5 } & $\begin{array}{c}\text { Density } \rho \\
{\left[\mathrm{kg} / \mathrm{m}^{3}\right]}\end{array}$ & $\begin{array}{c}\text { Thermal Conductivity } \\
{[\mathrm{W} / \mathrm{mK}]}\end{array}$ & $\begin{array}{c}\text { Thermal Diffusivity } \\
{\left[\mathrm{m}^{2} / \mathrm{s}\right]}\end{array}$ & Heat Capacity [J/kgK] \\
\hline Air & 1.39 & 0.0257 & $1.6810^{-5}$ & 1099 \\
\hline Nanocomposite & \multicolumn{5}{|c|}{$1.5710^{-7}$} & 1131 \\
\hline Pure Epoxy [9] & 1130 & 0.19 & $3.1910^{-7}$ & 1103 \\
\hline $\begin{array}{c}5 \mathrm{wt} \% \\
\text { GnP/Epoxy [4] }\end{array}$ & 1136 & 0.41 & $5.0810^{-7}$ & \\
\hline $\begin{array}{c}10 \mathrm{wt} \% \\
\text { GnP/Epoxy [4] }\end{array}$ & 1142 & 0.64 & & \\
\hline
\end{tabular}

\subsection{Full-Field Thermal Diffusivity Mapping Validation}

The RVE thermal response was used to simulate the thermal diffusivity mapping of a specimen with air cavities. The thermal diffusivity field was split into two fields - one containing the pure nanocomposite and the other the cavities only. Each field was processed separately to obtain the nanocomposite and cavities volume fraction field. Next, from each field, the material properties $(\mathrm{K}, \mathrm{C}, \rho)$ distribution was created and finally combined in a single algebraic matrix to be used as an input data to the analysis. To calculate the thermal diffusivity mapping of the FE specimen, thermal conductivity was calculated for each node of the mid-plane, while the time-variant temperature of these nodes was stored to determine the thermal diffusivity.

The validation of numerical models can be achieved by employing the CEN / CWA (CEN Workshop Agreement) 16799 standard [10]. The methodology integrates full-field 
comparison results with experimental uncertainties into a robust and flexible validation technique which decides on the acceptance or not of a numerical simulation model [11]. This technique comprises numerical simulations, full-field experimental results, data compaction using image analysis techniques and decision on the acceptance of the model based on the comparison of the deviation between the numerical and experimental results and the experimental uncertainties. Any data field I(x,y) can be fully described by a vector containing a small number (a few decades) of orthogonal moments (Zernike, Chebyshev, Fourier, Legendre, etc). As the reconstruction of an image using an infinite number of shape descriptors is not practically possible, an approximate reconstruction may be achieved by keeping only the moments and their relative shape polynomials of the higher value, while the remaining terms are discarded. An example of image reconstruction is presented in Fig. 4, where the region (i) of the experimental thermal diffusivity map (as presented in Fig. 6(i)) is reconstructed with Zernike moments. The accuracy of the shape moment approximation can be evaluated using different expressions. The most common equations are the Normalised Root Mean Square (NRMS) error (1) [12] and the Correlation Coefficient (2) [13], where $\mathrm{I}(\mathrm{x}, \mathrm{y})$ and $\hat{I}(x, y)$ are the original and the reconstructed fields, respectively, and $\overline{\hat{I}}=\frac{\iint_{\Omega} \hat{I} d A}{\iint_{\Omega} d A}, \bar{I}=\frac{\iint_{\Omega} I d A}{\iint_{\Omega} d A}$.
$e=\sqrt{\frac{\sqrt{\iint_{D}|I(x, y)-\hat{I}(x, y)|^{2} d x d y}}{\iiint_{D}|I(x, y)|^{2} d x d y}}$
$\operatorname{corr}(\hat{I}, I)=\frac{\iint_{\Omega}(\hat{I}-\overline{\hat{I}})(I-\hat{I}) d A}{\sqrt{\left[\iint_{\Omega}(\hat{I}-\overline{\hat{I}})^{2} d A\right]}\left[\iint_{\Omega}(I-\bar{I})^{2} d A\right]}$

The experimental and numerical thermal diffusivity field was reconstructed with Zernike polynomial. A large number of Zernike polynomials (400) has been used, and in both cases, the reconstruction error (NRMS) was around $10 \%$. Moreover, the image reconstruction with Zernike polynomials is characterised by smoothing the field with noise removal. However, in this case, Zernike decomposition resulted in increasing the peak values, instead of decreasing them, therefore de-noising could not be achieved. Such phenomenon is more evident in the reconstruction of the experimental field compared to the numerical one. This could be attributed to the fact that polynomials are able to reconstruct fields whose spatial evolution is uniform and in form of shape patterns. For this reason, the field was divided into smaller areas with the simplest contour possible. These areas were reconstructed and compared on the basis of the linear correlation plot. The reconstruction error of each region is graphically presented in Fig. 5. It could be noted that the reconstruction efficiency is rapidly improved with significantly lower errors. The experimental thermal diffusivity mapping of the $10 \mathrm{wt} \% \mathrm{GnP} /$ epoxy specimen along with the areas studied under full-field validation are shown in Fig. 6(i). The selected areas show a single surface cavity or regions of pure nanocomposite without defects. The fields are compared on the linear correlation plot (Fig. 6(ii)) and all the points were found to lie in the area defined by the experimental uncertainties $u= \pm 0.3510^{-7} \mathrm{~m}^{2} / \mathrm{s}$, proving the method is validated. 

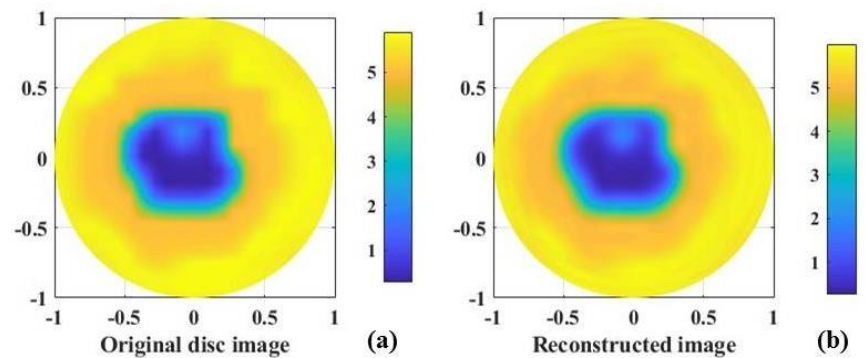

Fig. 4. (a)Original disc image and (b) its reconstructed one with Zernike moments (Region i of Fig. 6(i)).

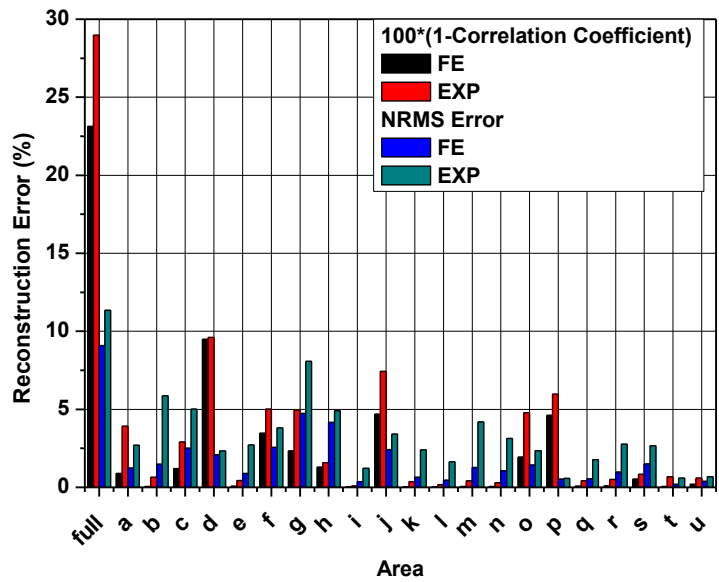

Fig. 5. Reconstruction errors of the numerical and experimental field for every studied area using the correlation coefficient and the NRMS error.
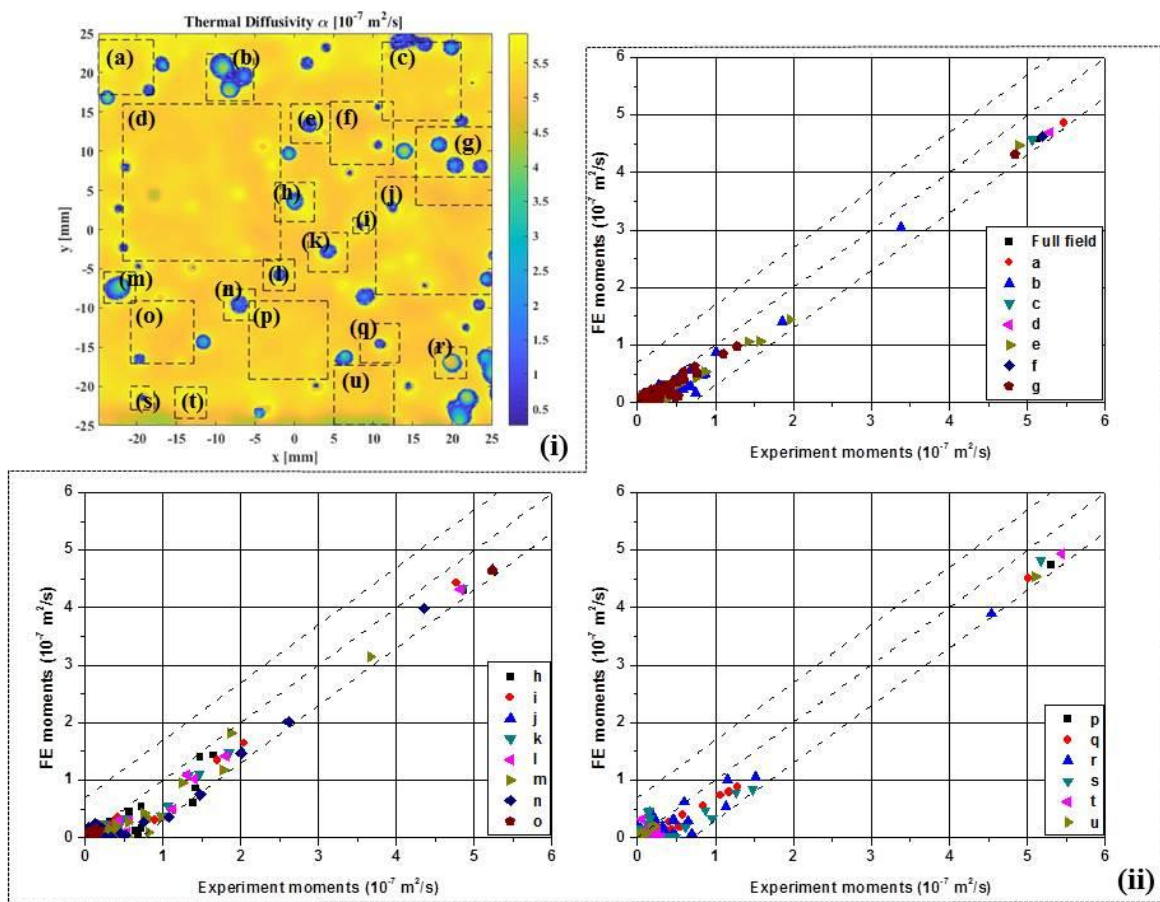

Fig. 6. (i) Experimental thermal diffusivity mapping of $10 \mathrm{wt} \% \mathrm{GnP} /$ epoxy with the numbering of areas studied under full field validation and (ii) linear correlation plots of every studied area. 


\section{Conclusions}

In this research work a $10 \mathrm{wt} \% \mathrm{GnP} /$ epoxy nanocomposite specimen with air cavities was prepared. These voids were the result of unsuccessful degasification and air trapped during the curing process. The specimen was subjected to infrared thermography for measuring thermal diffusivity mapping. The cavities were detected and mapped by processing the temperature fields. The average void volume fraction was estimated to $3.07 \%$ and average field thermal diffusivity of $5.0310^{-7} \mathrm{~m}^{2} / \mathrm{s}$. The effect of air bubbles to the thermal response of nanocomposites was studied through a multi-scale scheme including an RVE and a macro-scale specimen. In the RVE, the effect of a single cavity to the local response was studied. The macro-scale specimen was built for the simulation of the experimental thermal diffusivity mapping. The measured experimental thermal diffusivity was found to be in good agreement with the RVE model. The thermal diffusivity mapping was simulated and compared to the experimental one through the CEN/CWA 16799 standard. When the full field was reconstructed, the reconstruction error was high and the Zernike moments were not able to represent efficiently the map. Therefore, the field was divided to smaller simpler areas, then reconstructed and compared separately. As a result, the reconstruction efficiency was rapidly improved. The examined areas were compared in the correlation plots with all points lying in the validation band with an uncertainty of $0.3510^{-7} \mathrm{~m}^{2} / \mathrm{s}$. It can be said that the proposed methodology is validated and able to simulate the thermal response of nanomaterials containing fabrication defects.

\section{References}

1. A. Manta, M. Gresil, C. Soutis, Appl. Compos. Mater., 24, 2, 281-300, (2017)

2. A. Manta, M. Gresil, C. Soutis, Compos. Struct., 192, 452-459, (2018)

3. A. Manta, M. Gresil, C. Soutis, in Graphene2017, (2017)

4. A. Manta, M. Gresil, C. Soutis, AIP Conference Proceedings, 1932, 1, 20001, (2018).

5. XGSciences, "xGnP Technical Data Sheet," 2013

6. C. Huntsman, "Araldite LY 564 / Aradur 2954 Technical Datasheet."

7. "ASTM E1461-13. Standard test method for thermal diffusivity by the flash method," ASTM, 2001

8. W. J. Parker, R. J. Jenkins, C. P. Butler, G. L. Abbott, J. Appl. Phys., 32, 9, 1679, (1961)

9. M. Gresil, Z. Wang, Q.-A. Poutrel, C. Soutis, Sci. Rep., 7, 1, 5536, (2017)

10. E. Hack, E. Patterson, R. Burguete, A. Davighi, M. Feligiotti, A. Ihle, G. Lampeas, J. Mottershead, A. Pipino, H. Schubach, T. Siebert, W. Wang, , M. Whelan, "CEN/CWA16799: Validation of computational solid mechanics models." Brussels: Comité Européen de Normalisation, (2014)

11. G. Lampeas, V. Pasialis, X. Lin, and E. A. Patterson, Simul. Model. Pract. Theory, 52, 92-107, (2015)

12. S. X. Liao,M. Pawlak, Computer Vision - ACCV'98. ACCV 1998. Lecture Notes in Computer Science, Springer, Berlin, Heidelberg, (1997), 394-401, (1997)

13. W. Wang, J. E. Mottershead, C. M. Sebastian, E. A. Patterson, Int. J. Solids Struct., 48, 1644-1657, (2011) 